\title{
ANALISIS FENOTIPICO DEL COMPLEX Trichophyton rubrum EN LOS MEDIOS DE SABOURAUD, LACTRIMEL, Y LA TECNICA DE FISCHER \& KANE: UNA EXPERIENCIA REGIONAL
}

\author{
(Phenotipic analysis of the Trichophyton rubrum complex in \\ Sabouraud's dextrose agar, Lactrimel agar and the Fischer \& Kane technique: \\ A regional experience). \\ *M. Alicia Toro;**Dunny Casanova \& *Eduardo Piontelli \\ *Universidad de Valparaíso, Escuela de Medicina \\ Cátedra de micología, Casilla 92 V Valparaíso, Chile. e-mail <maria.toro@ uv.cl > \\ **Universidad de Valparaíso, Escuela de Medicina, Departamento de Salud Pública
}

Palabras clave: Sabouraud dextrosa agar, Lactrimel agar, medio Fischer \& Kane, complex Trichophyton rubrum. Key words: Sabouraud's dextrose agar, Lactrimel agar, Fischer \& Kane media, Trichophyton rubrum complex

\section{RESUMEN}

Con el propósito de comparar el rendimiento y la eficiencia de los medios utilizados de rutina en el laboratorio de Micología para la identificación de las especies que integran el complex T. rubrum presentes en la $\mathrm{V}$ Región, Chile, seleccionamos: Agar glucosa Sabouraud (SDA), Agar Lactrimel (AL) y el medio sólido leche, glucosa, adicionado con el indicador de púrpura de bromocresol (Fischer \& Kane, 1971) (BCP-MS-G). Estos fueron probados en 304 cepas consideradas como $\mathbf{T}$. rubrum en el medio SDA, provenientes de pacientes dermatológicos. Los parámetros que se consideraron fueron rango de crecimiento, pigmentación al reverso de las colonias y presencia de fructificaciones. Las variedades del complex identificadas fueron T. rubrum var. algodonosa $(72,4 \%)$ y T. rubrum var. granular (14,5\%) además de integrantes del complex $\boldsymbol{T}$. mentagrophytes $(13,2 \%)$ que no se consideraron en el estudio. La zona anatómica más afectada fueron las uñas de los pies (80,7\%). La fructificación en los 3 medios fue considerada como el «gold standard» y en base a este criterio se estableció su sensibilidad y especificidad, obteniéndose para la var. granular una especificidad de un 100, 98,6 y 83,9\% en BCP-MS-G, AL y SDA, respectivamente. Para la var. algodonosa, la mayor sensibilidad se obtuvo en BCP-MS-G (71,9\%), pero con una nula especificidad, por lo que se sugiere considerar el rango de crecimiento limitado a los 7 días y la pigmentación al reverso de la colonia en este medio. La identificación fue complementada con la prueba de la ureasa, que fue 100\% negativa para todo el complex T. rubrum. Se sugiere el uso del medio de Fischer \& Kane en conjunto con $A L y$ SDA para diferenciar las variedades de este complex.

\section{ABSTRACT}

The following media Sabouraud's dextrose agar (SDA), Lactrimel Agar and BCP-Milk solids glucose agar (Fischer \& Kane, 1971) (BCP-MS-G) were selected to evaluate the performance and the efficiency of the routine media used in mycological laboratory with the purpose of identifying the species that conformed T. rubrum complex in the V Region of Chile. These were tested in 304 strains considered as T. rubrum in the SDA media, obtained from dermatological patients. The following parameters were used: the radial growth of the colonies, the pigmentation at the back of the plates and the presence of fructification. T. rubrum variety downy $(72,4 \%$ and $\mathbf{T}$. rubrum variety granular $(14,5 \%)$ were the only variants identified and T. mentagrophytes (Robin) Blanchard $(13,2 \%)$, that were not consider in this study. The mosts affected anatomic zones were the toe nails (68,9\%). In the three media used, we considered the presence of fructifications as the «gold standard» and based on this criteria we evaluated the sensibility and specificity of them. For the variant granular we obtained a specificity of 100 , 98,6 y 83,9\% en BCP-MS-G, AL y SDA, respectively. For the variant downy, the greatest specificity was obtained in BCP-MS-G 71,9\%,but with a null specificity, a cause 
of this situation we propose to considerer the restricted radial growth of colonies on the seventh day and the red pigmentation at the back of the plates in this media. The identification of the complex was complemented by the urea test, that was negative for the whole complex. The use of Fischer and Kane media in combination with $A L$ and SDA is suggested for the differentiation of the varieties of this complex.

\section{INTRODUCCION}

Los dermatofitos constituyen un grupo de hongos relacionados entre sí, capaces de invadir los tejidos queratinizados del hombre y de los animales produciendo cuadros clínicos denominados generalmente dermatofitosis.

Según su nicho ecológico (reservorio, suelo, animal u hombre) se les califica como geofílicos, zoofílicos y antropofílicos, sin embargo, es necesario destacar que algunos de los integrantes de cualquiera de estos tres grupos ecológicos bajo condiciones favorables, son potencialmente capaces de producir enfermedad en el hombre (Gräser et al., 1999). Las diversas especies de dermatofitos no se aíslan con la misma frecuencia ya que existen condiciones climáticas, geográficas socioeconómicas y otras que originan cambios en los patrones de distribución de estos agentes etiológicos. Su identificación rutinaria en el laboratorio de Micología se basa fundamentalmente en criterios morfológicos macro-microscópicos relacionados con sus estados anamorfos, que incluyen los géneros Microsporum, Trichophyton y Epidermophyton. Taxonómicamente se agrupan dentro de los Ascomycota, (Eurotiomycetes, Onygenales, Arthrodermataceae). Actualmente la familia incluye 3 géneros donde Arthroderma es el teleomorfo de Microsporum y Trichophyton (Weitzman et al., 1986; Gräser et al., 1999; Simpanya, 2000; Ericsson, 2006).

Los criterios morfológicos muestran una sustancial variación y pleomorfismo dentro de los taxa establecidos, por lo que se han adicionado pruebas fisiológicas y moleculares (Brasch et al., 1991; Rezaie et al., 1999; Harmsen et al., 1999; Gräser et al., 1999; Gräser et al., 2000; Simpanya, 2000; Gupta et al., 2001; Ellis, 2004).

Entre las especies que se distribuyen en forma regular se indican en la literatura nacional: T. rubrum, M. canis (Bodin) Bodin y T. mentagrophytes (Robin) Blanchard (Piontelli et al., 1991; Zaror et al., 1995; Díaz et al., 1995; Pérez et al., 2001; Zepgig et al., 2004). Considerando que el cultivo fúngico continúa siendo el «gold standard» del diagnóstico micológico, creemos necesario evaluar la efectividad de los medios de rutina que permitan en un tiempo no mayor de 12 a 14 días la diferenciación de las variedades del complex $\boldsymbol{T}$. rubrum en una zona geográfica limitada, seleccionando para ello los siguientes medios: Agar glucosa Sabouraud (SDA), Agar Lactrimel (AL), comparándolo con el medio Agar glucosa y leche, púrpura de bromocresol (PBC-MS-G) propuesto por Fischer \& Kane (1971).

\section{MATERIALES Y METODOS}

1.- Especímenes seleccionados. En el curso del período Agosto 2004 a Septiembre del 2005 se obtuvieron de 304 cepas clasificadas en SDA como T. rubrum desde pacientes derivados de consultorios dermatológicos públicos y privados enviados al laboratorio de micología de nuestra Escuela de Medicina. Las cepas obtenidas fueron aisladas de diferentes localizaciones anatómicas (uñas, pies-manos y piel-cuerpo). Los aislados al estado puro (sin bacterias ni levaduras) fueron sometidas a las siguientes etapas:

a) Siembra de las muestras en SDA, AL y PBCMS-G en placas de $5 \mathrm{~cm}$ en duplicado, incubados los 2 primeros por 12 días y el último por 10 días a $27^{\circ} \mathrm{C}$. El inóculo se obtuvó mediante una cuchareta de $1 \mathrm{~mm}$ de diámetro tomado del borde de la colonia.

b) De las colonias obtenidas en los 3 medios se procedió a aplicar los siguientes criterios morfofisiológicos: Rango de crecimiento: considerándose restringido menor o igual a $10 \mathrm{~mm}$ y sobre $11 \mathrm{~mm}$ no restringido. Morfología de la colonia (plana, solevantada, regular o irregularmente plegada), textura (glabra, levaduriforme, pulverulenta, granular, aterciopelada, lanosa), pigmentación al reverso de la colonia. Esta última se diferenció en rojo (1), otras tonalidades: amarillo café, amarillo naranja (2) y ausencia de pigmento (3).

2.- Morfología microscópica, se registró: a) la ausencia de microconidios en el cultivo, b) presencia de microconidios (escasos, abundantes y presencia de micro y macroconidios).

3.- Detección de ureasa: Todas las cepas fueron sembradas e incubadas a $27^{\circ} \mathrm{C}$ durante 10 días en agar urea de Christensen para la detección de la ureasa a los 7 días.

La validez de los medios utilizados se realizó aplicando los conceptos de sensibilidad y especificidad, considerando como «gold standard» la fructificación de las cepas estudiadas.

\section{RESULTADOS}

Del análisis de las 304 muestras procesadas se detectó la siguiente frecuencia de aislamiento por agente causal, correspondientes a T. rubrum var. algodonosa 220 cepas (72,4\%), $\boldsymbol{T}$. rubrum var. granular 44 (14,5\%) y a T. mentagrophytes, $40(13,2 \%)$ debido a que el 


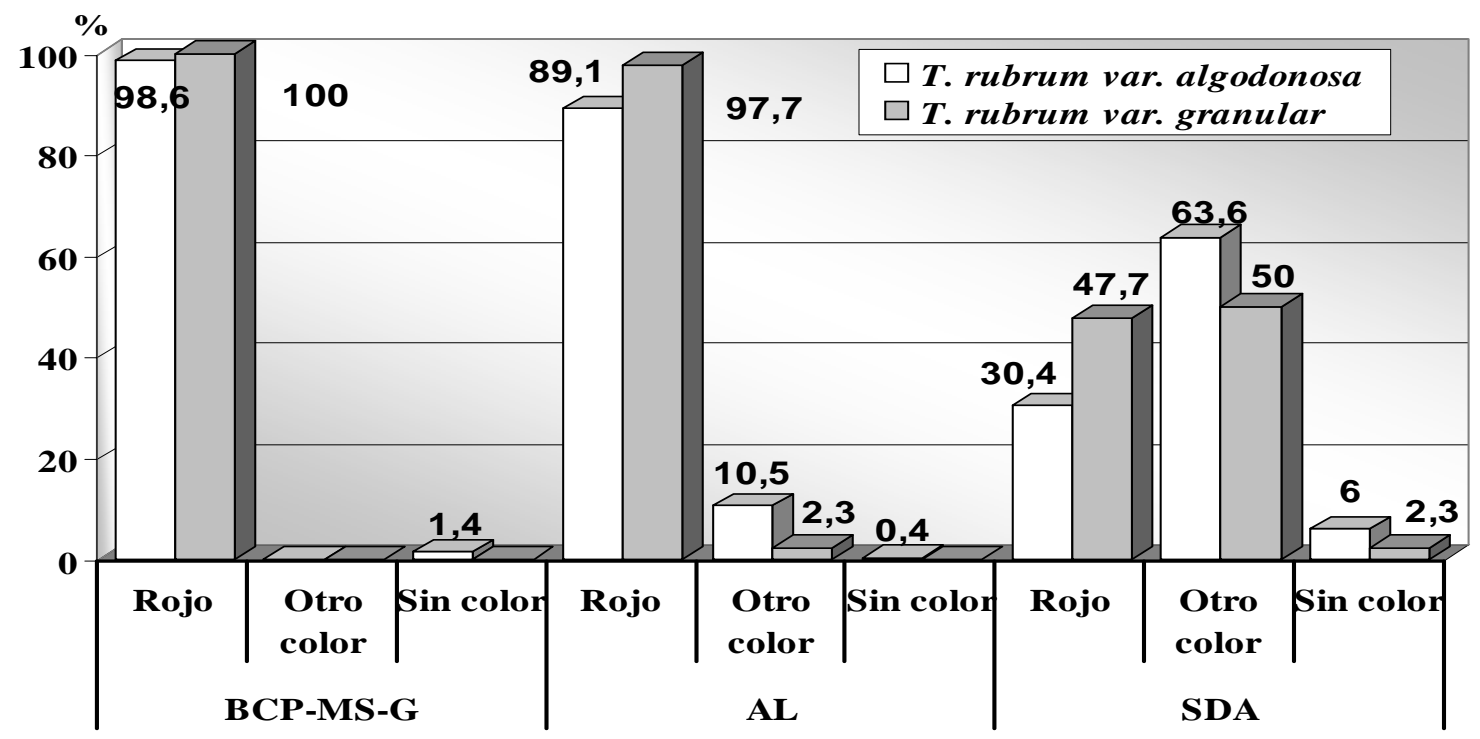

Coloración al reverso de la colonia según medio de cutivo

Figura 1.- Presencia de pigmentación (\%) al reverso de las colonias en los tres medios sobre el total del complex T.rubrum y las dos variedades detectadas.

desarrollo de estas últimas no fue claramente diferenciado en SDA (por lo tanto se consideraron solo 264 cepas ).

De las muestras analizadas (182/264) el 68,5 \%, afectaron las uñas de los pies, de las cuales 151 (83\%) correspondieron a T. rubrum var. algodonosa, 31 (17\%) a T. rubrum var. granular. Otras áreas corporales afectadas con menor frecuencia por T. rubrum var. algodonosa fueron piel cuerpo con un 20 \% y uñas manos 8,3 \%, mientras para la var. granular tuvo bajo registros en piel $(1,8 \%)$ y uñas manos $(1,8 \%)$.

Respecto al rango de crecimiento de las colonias expresados en el diámetro a los 12 días, la media aritmética obtenida en el medio BCP-MS-G, AL y SDA fue de 12,0, 14,5 y $18,2 \mathrm{~mm}$, repectivamente. El crecimiento reducido de las colonias, menor o igual de $10 \mathrm{~mm}$ en el medio BCPMS-G se apreció a los 7 días.

La pigmentación roja al reverso de las colonias (uno de los criterios para identificar el complex T. rubrum) en el medio BCP-MS-G, para la var. algodonosa se manifestó en un $98,6 \%$, en un $89,1 \%$ en AL y sólo un $30,5 \%$ en
SDA. En cambio para la var. granular la pigmentación fue prácticamente de un $100 \%$ en los 2 primeros medios mientras en SDA fue levemente menor al 50\% (Figura 1).

La fructificación de las especies del complex en los medios, ya sea por la ausencia o presencia de conidios, alcanzó su mayor expresión tanto en el medio BCP-MS$\mathrm{G}$ como AL siendo para la var. algodonosa de un 51\% y un $35 \%$ y de un $100 \%$ y $96 \%$ en la granular respectivamente (Tabla 1).

La morfología macroscópica de las colonias presentó principalmente 2 texturas, la algodonosa y la granulosa, las que fueron un complemento en la diferenciación de las 2 variedades del complex.

La mayor producción de macroconidios se observó sólo en la var. granular de los medios AL (25\%) y BCPMS-G (13,6\%). En SDA fue negativo para ambas variedades.

La evaluación de los medios de cultivo en función de la producción o ausencia de fructificación de las especies del complex, determinó una especificidad para

Tabla 2.- Frecuencia y porcentaje de fructificaciones de las especies del complex T. rubrum en BCP- MS-G, AL y AGS.

\begin{tabular}{|c|c|c|c|c|c|c|c|c|c|c|c|c|c|}
\hline \multicolumn{2}{|c|}{ Medios de Cultivo } & \multicolumn{4}{|c|}{ BCP-MS-G } & \multicolumn{4}{|c|}{ AL } & \multicolumn{4}{|c|}{ SDA } \\
\hline & $\mathbf{N}$ & A & $\%$ & $\mathbf{P}$ & $\%$ & A & $\%$ & $\mathbf{P}$ & $\%$ & A & $\%$ & $\mathbf{P}$ & $\%$ \\
\hline $\begin{array}{l}\text { T. rubrum var. } \\
\text { algodonosa }\end{array}$ & 220 & 107 & 49 & 113 & 51 & 144 & 66 & 76 & 35 & 212 & 96,4 & 8 & 3,6 \\
\hline $\begin{array}{l}\text { T.rubrum var. } \\
\text { granular }\end{array}$ & 44 & 0 & 100 & 44 & 100 & 2 & 4,5 & 42 & 96 & 41 & 93,2 & 3 & 6,8 \\
\hline
\end{tabular}




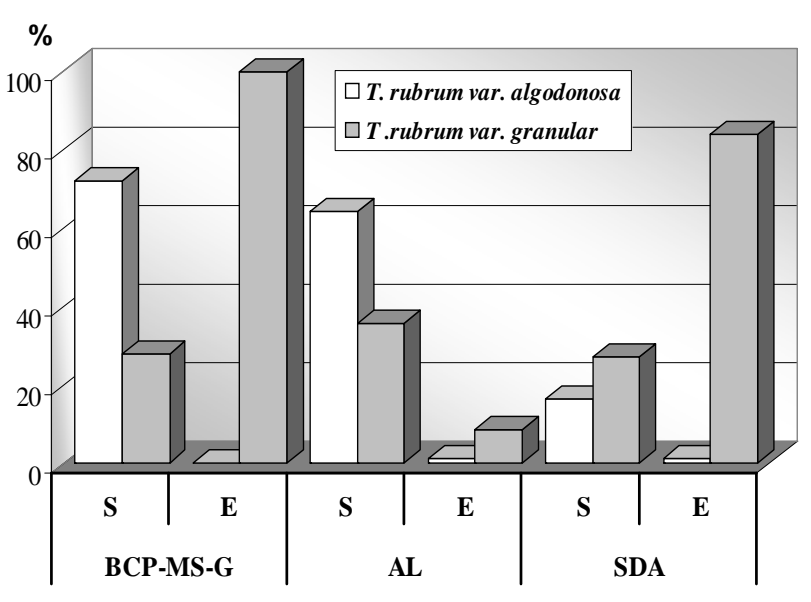

Sensibilidad (S) y Especificidad (E) de los medios utilizados

\section{Figura 2.- Sensibilidad y Especificidad de los medios BCP-MS-G, AL y AGS.}

la variedad granular de un $100 \%$ en el medio BCP-MSG, seguido por el AL (98,6\%) y SDA (83,9\%). Para la variedad algodonosa la sensibilidad en el medio BCP-MSG fue de un $71,9 \%$ con una nula especificidad (Figura 2).

Las 264 cepas correspondientes al complex $\boldsymbol{T}$. rubrum fueron ureasa negativas.

\section{DISCUSION}

Los dermatofitos constituyen un grupo de hongos conocidos por causar infecciones superficiales en el hombre y en los animales. Usualmente se visualizan en los cultivos en sus formas conidiales (anamorfos) y se clasifican en los géneros Epidermophyton, Keratinomyces, Microsporum y Trichophyton. Los estados sexuales (teleomorfos), cuando están presentes integran la familia Arthrodermataceae, dentro de los Onygenales (Eurotiomycetes). Tradicionalmente los teleomorfos de Microsporum se incluían en Nannizzia y aquellos pertenecientes a Chrysosporium, Keratinomyces y Trichophyton en Arthroderma, actualmente el género Nannizzia ha sido incorporado al género Arthroderma (Weitzman et al., 1986). Según su nicho ecológico se les designa como geofílicos, zoofílicos o antropofílicos, sin embargo los miembros de estos tres grupos son considerados como potencialmente patógenos (Kane et al., 1997).

La identificación de las especies del complex $\boldsymbol{T}$. rubrum (Castell.) Semon, no es fácil como consta en diversos estudios realizados dentro de este contexto, en especial debido a su pleomorfismo y variabilidad de sus cultivos complicando su identificación y diferenciación (Georg, 1953; Mc Donough, 1960; Borelli, 1962; Rebell \& Taplin 1970; Fischer \& Kane, 1971; Vidotto et al,1983;
Weitzman \& Summerbell, 1995; Gräser et al., 1999; Singh \& Benna, 2003).Básicamente su identificación se apoya al igual que para las otras especies de dermatofitos en las características morfológicas y fisiológicas de las colonias, entre ellas la pigmentación al reverso de éstas, y numerosos medios de cultivo han sido propuestos y modificados en un intento de mejorar su diagnóstico con criterios de fácil aplicación en la identificación de las especies que integran el complex T. rubrum (Piqué et al., 2002; Singh \& Beena, 2003).

Young (1972), investigó la estabilidad morfológica de las cepas incluidas en la especie críptica $T$. rubrum estableciendo 7 formas distintivas que difieren en sus patrones morfológicos: algodonoso, granular, disgónico, melanoide, africano, hiperpigmentado y rodhainii. Ellis (2004), comenta que la mayoría de las formas y variedades del complex T. rubrum descritas, genera una cierta controversia entre los especialistas y prácticamente se diferencian y observan mayoritariamente sólo dos tipos morfológicos, el algodonoso y el granular. En concordancia con el comentario de Ellis, el medio BCP-MS-G utilizado nos permitió detectar estas dos variedades.

Gräser et al. (2000), señalan que las especies de dermatofitos más prevalentes en el mundo corresponden al complex $\boldsymbol{T}$. rubrum. La literatura internacional señala que la población norteamericana se ve afectada entre un 6 a un $13 \%$ por este agente causal (Gupta \& Summerbell, 2001). Se ha sugerido que este complex ha evolucionado a partir del siglo XIX como agente causal de tinea corporis crónica, diseminándose por lo diferentes continentes como agente etiológico de onicomicosis y tinea pedis, además destacan que las especies que integran este complex, originalmente descritas por Young (1972), raramente son aisladas como agentes de dermatomicosis, excepto las variedades algodonosa y granular (Ellis, 2004). Gräser et al. (1999), analizaron 100 cepas del complex T. rubrum, incluyendo las variedades fenotípicas, no detectaron ninguna variabilidad del DNA entre las cepas estudiadas. Posteriormente Gräser et al. (2000), mediante una combinación de técnicas fenotípicas y moleculares, donde las características morfológicas y fisiológicas fueron comparadas con secuenciación de ITS, PCR, «fingerprints» $\mathrm{y}$ análisis de longitud de fragmentos polimóficos (AFLP), reclasificaron o establecieron sinonimías con respecto a las dos clade monofiléticas diferenciadas por su secuencia de ITS: 1 . T. violaceum que es el taxon más antiguo identificado y clade $2 . \boldsymbol{T}$. rubrum y T. megninii, ambos estrechamente relacionados y coespecíficos. Estos resultados fueron comparados con los datos convencionales, destacándo que el origen de este complex es exclusivamente antropofílico. Ohst et al. (2004), aplicando la técnica de los microsatélites como marcadores, detectaron cierta asociación entre el origen 
geográfico de los aislados estudiados: estos autores sugieren que el complex $\boldsymbol{T}$. rubrum es de origen africano, a partir del cual ha emergido un nuevo genotipo (B) en el Asia con su consiguiente diseminación por Europa y Estados Unidos de Norteamérica

Con el propósito de comparar el rendimiento y la eficiencia de los medios utilizados de rutina por otros investigadores, seleccionamos el Agar glucosa Sabouraud (SDA) (Ajello et al. 1966), Agar Lactricmel (AL) (Borelli, 1962) y el medio sólido leche, glucosa, adicionado con el indicador de púrpura de bromocresol (Fischer \& Kane, 1971) (BCP-MS-G).

De las variables seleccionadas, consideramos en primer lugar el crecimiento radial de las colonias de $\boldsymbol{T}$. rubrum, en el medio (BCP-MS-G), por su patrón de crecimiento restringido. El criterio primario aplicado por Fischer $\&$ Kane diferencia las especies de $\boldsymbol{T}$. rubrum por su crecimiento reducido con respecto a las colonias de $\boldsymbol{T}$. mentagrophytes, asociado a la participación de la actividad enzimática de las proteasas de las especies de $\boldsymbol{T}$. rubrum, iniciada por la liberación de los iones de amonio provenientes de la caseína de la leche y su posterior represión metabólica por la incorporación de la glucosa. Esta represión finaliza aproximadamente a las dos semanas, tiempo durante el cual se ha consumido la glucosa, es decir, al suprimirse la amonificación; el crecimiento radial se restringe durante los primeros 10 días a $27^{\circ} \mathrm{C}$. Esta situación se visualiza gracias a la acción del indicador púrpura de bromocresol, donde T. mentagrophytes da origen no sólo a una colonia extensa y profusa dentro de 4 días sino que además el medio que tiene un color azul cielo vira a violeta-púrpura indicando un $\mathrm{pH}$ alcalino; en cambio las colonias de $\mathbf{T}$. rubrum mantienen el color inicial del medio, un $\mathrm{pH}$ neutro hasta después de transcurridos 10 a 14 días (Summerbell et al., 1988; Weitzman \& Summerbell, 1995). Las medias aritméticas del diámetro de las colonias en los tres medios, nos permitió apreciar este patrón restringido a los 7 días

En segundo lugar, la presencia del pigmento rojo al reverso de la colonia es distintiva para el complex $\boldsymbol{T}$. rubrum y es un parámetro que permite diferenciar las especies en ausencia de fructificación, obteniéndose los mejores resultados en BCP-MS-G y AL. Entre las características fenotípicas de estos agentes, el pigmento es citado y asociado a procesos infecciosos junto a la presencia de artroconidios. La producción de estos metabolitos secundarios observados como compuestos coloreados en los cultivos, cumplirían una acción preventiva limitando la existencia de competidores bacterianos presentes en la piel y en las uñas (Summerbell, 2000). La glucosa, estimula la incorporación de oxígeno, actividad asociada a la enzima deshidrogenasa adenina dinucleótido fosfato (NADP), que condiciona la actividad respiratoria endógena de los microconidios que se incrementan al disminuir la enzima, por el contrario cuando ésta aumenta su actividad repercute en la formación de macroconidios (Mc Bride \& Stock, 1966).

El SDA ha sido considerado como el mejor medio estandarizado para aislamientos primarios de dermatofitos (Calvo et al., 1986; Singh \& Beena, 2003), sin embargo, comparado con los otros dos medios, observamos que no discrimina entre la variedad de pigmentos que pueden desarrollarse al reverso de la colonia para las especies del complex. Taplin et al. (1969), evalúan el medio DTM estableciendo su eficiencia con respecto al SDA $(p=0,05)$. El principio de acción de este medio es semejante al BCPMS-G pues se fundamenta en la alcalinidad desarrollada por los dermatofitos, los cuales promueven un cambio de color rojo, que es registrado por el indicador rojo fenol, no obstante algunos hongos apatógenos como $T$. terrestre pueden inducir este cambio, como también otras especies de dermatofitos contaminados con bacterias, dando reacciones falsamente negativas. Entre sus ventajas se destaca que es útil para un diagnóstico presuntivo de dermatofitos a los dos días, pero impide la visualización del pigmento al reverso de la colonia, una característica importante en la identificación de este grupo (Weitzman \& Summerbell, 1995; Pariser \& Opper, 2002; Singh \& Benna, 2003).

Estudios previos realizados por Fischer \& Kane (1971), destacan la dificultad de obtener cultivos puros de las muestras dermatolológicas analizadas, donde la contaminación bacteriana no es prevenida por el cloranfenicol, aunque las bacterias sean sensibles a este antimicrobiano, ya que al inocular el raspado de piel, cuero cabelludo, u otras muestras, no todas las partes de éstas se ponen en contacto con la superficie del medio. O bien a medida que el hongo desarrolla su micelio aéreo, las bacterias pueden crecer en las gotas formadas por la humedad que se acumula sobre los filamentos fúngicos, lo cual es fácilmente detectable en BCP-MS-G. Es interesante destacar la opinión de otros autores que han utilizado este medio, quienes señalan que la identificación de las diversas especies de dermatofitos no suele ser enmascarada o interferida por la contaminación bacteriana o fúngica y que por lo general, son las causales de las formas aberrantes de la especie $\mathbf{T}$. rubrum o de la pérdida del pigmento (Summerbell et al. 1988).

Según Calvo (1986), el medio SDA es ideal para el aislamiento primario de la especie $\boldsymbol{T}$. mentagrophytes, situación que hemos observado en nuestra investigación donde los tres medios estudiados permiten una buena identificación y diferenciación de los integrantes de este complex (datos no publicados).

La alta prevalencia de las lesiones en las uñas de los pies $(80,7 \%)$ se debe principalmente por el estilo de 
vida del hospedador humano, en particular por el uso del zapato cerrado que favorece el alza de la temperatura y la humedad de la piel (Gräser et al., 2000). Esto concuerda con los resultados de investigadores nacionales quienes han señalado que las lesiones más frecuentes originadas por T. rubrum son en las uñas de los pies (Piontelli et al., 1991; Zaror et al., 1995).

Nuestros resultados demuestran la presencia de dos variedades de $\boldsymbol{T}$. rubrun en nuestra región, una experiencia que necesita repetirse en otras zonas geográficas del país y confirman las conclusiones establecidas por Summerbell et al., (1988). Podemos añadir que las dificultades mencionadas para el aislamiento, identificación y diferenciación de las variedades que integran el complex T. rubrum, podrían reducirse usando el medio BCP-MS-G o el AL que ofrecen una alternativa confiable y segura, con un tiempo de incubación entre 10 a 12 días.

\section{REFERENCIAS}

Ajello, L.; Georg, L.K.; Kaplan, W.; Kaufman, L. (1966). Laboratory Manual for Medical Mycology. Department of Health Service, Communicable Disease Centre, Atlanta, Georgia.

Borelli, D. (1962). Medios caseros para micología. Arch. Venez. Med. Trop. Paracet. Med. 4:301-310

Brasch, J.; Britta-Sigrid, M.;Christophers, E. (1991). Enzyme release by Trichophyton rubrum depends on nutritional conditions. Mykoses 34:365-368

Calvo, M.A.; Trape, J.; Abarca, L.;Cabañes, J.F.; Calvo, R.M.; Bruguera, T. (1986). Variability of biochemical characteristics in strain of Trichophyton mentagrophytes. Mycopathologia 93:137-139

Díaz, Y. M.; Díaz, M.C.; Garreaud de Mainvilliers, G. C. (1995). Frecuencia de dermatofitos en pacientes hospitalizados. Rev. Chil. Infectol. 12:141-145

Ellis, D. (2004). Laboratory Methods. Mycology on line, Fungal descriptions, dermatophytes, Trichophyton. CRICOS Provider number 00123M School of Molecular \& Biomedical Science. The University of Adelaide. Australia.

Ericsson, O.E. (2006). Outline of Ascomycota. Myconet 12:1-82

Fischer, J.B. \& Kane, J. (1971). The detection of contamination in Trichophyton rubrum and Trichophyton mentagrophytes. Mycopath. et Mycol. Appl. 43:169-180

Georg, L.K. (1953). Use of cicloheximide mediun for isolation of dermatophytes from clinical lesions. Arch. Dermatol. Syphyl. 63:355361

Gräser, Y.; El Far, M.; Vilgalis,R.; Kuijpers, A.F.A.; De Hoog, G.S.; Presber, W.; Tietz, H.J. (1999). Phylogeny and Taxonomy of the Family Arthrodermataceae (dermatophytes) using sequence analysis of the ribosomal ITS region. Medical Mycology 37:105-114

Gräser, Y.;Kuijpers, A.F.A.; Presber, W.; De Hoog, G.S. ( 2000). Molecular taxonomy of Trichophyton rubrum complex. Journal of
Clinical Microbiology 38:3329-3336

Gupta, K.A.; Kohli, Y.; Summerbell, R.C. (2001). Variation in restriction fragment length polymorphisms among serial isolates from patients with Trichophyton rubrum infections. Journal of Clinical Microbiology 39:3260-3266

Harmsen,D.; Schwinn, A.; Brocker, E.B.; Frosch, M. (1999). Molecular differentiation of dermatophyte fungi. Mykosen 42: 67-70

Kane, J.; Summerbell, R.; Sigler, L. Krajden, S.; Land, G. (1997). Laboratory Handbook of Dermatophytes. Star Publishing, Belmont, USA.

Mc Bride, B. C. \& Stock, J. J. (1966). Oxidative metabolism of dermatophytes. Applied Microbiology 14:973-978

Mc.Donoungh, E.S. (1960). Growth of dimorphic human pathogenic fungi on media containing cicloheximide and chloranphenicol. Micopath. et Micol. Appl. 13:113-120

Ohst, T.; de Hoog, S.; Prisber, W.; Stavrakieva, V.; Gräser, Y. (2004). Origins of microsatellite diversity in the Trichophyton rubrum - T. violaceum Clade (Dermatophytes) Journal of Clinical Microbiology 42: 4444- 4448

Oyeka, C.A. (2000). Trichophyton mentagrophytes a keratinophilic fungi. En Biology of dermatophytes and other keratinolitic fungi. Rev. Iberoamericana de Micología, Editores Kushwaha \& J. Guarro. pp.6065

Pariser D. \& Opper K. (2002). An in-office diagnostic procedure to detect dermatophytes in a nationwide study of onycomycosis patients. Managed Care, March:43-50

Park, Jin. Hee \& Choi,Won. Pil (2002). Biological characteristics of Trichophyton spp. and subtypes of Trichophyton mentagrophytes isolated from animals in Korea. Korean Journal of Veterinary Public Health 26:261-268

Pérez, B., M. \& Rivera, F. N. (2001). Agentes etiológicos de dermatomicosis aislados en los pacientes de la ciudad de Concepción y comunas circunvecinas: Chile 1998-1999. Rev. Chil.Tecnol. Med., 21:939-944

Piqué, E.; Fusté, R.; Copado, R.; Noguera J.; Ramis, P. (2002). Estudio de los dermatofitos en Lanzarote (1995-1999). Rev. Iberoamericana de Micología 19:165-168

Piontelli, E.; Toro, M.A.; Jara, D. (1991). Micosis superficiales en pacientes de servicios dermatológicos de la V Región: estudio de prevalencia en el período 1984-1989. Bol. Micol. 6:63-68

Rebell, G. \& Taplin, D. (1970). Dermatophytes their recognition and identification. University of Miami Press. Florida.

Rezaie, S.; Pourmojib, M.; Tschahler, E. (1999). Isolation of total RNA from dermatophytes. Mykoses 42:615-617

Shadomy, H.J. \& Philpot, C.M. (1980). Utilization of satandard laboratory methods in the laboratory diagnosis of problem dermatophytes. American Journal Clinical Pathology. 74:197-201

Singh,S. \& Beena P.M. (2003). Comparative study of different microscopic techniques and culture media for the isolation of dermatophytes. Indian Journal Medical Microbiology 21:21-24 
Simpanya, F. M. (2000). Dermatophytes: Their taxonomy, ecology and pathogenicity. En Biology of dermatophytes and other keratinolitic fungi. Rev. Iberoamericana de Micología, Editores Kushwaha \& J. Guarro.

Summerbell, R.C.; Rosenthal, S.A. \& Kane, J. (1988). Rapid method of differentiation of Trichophyton rubrum, Trichophyton mentagrophytes, and related dermatophyte species. Journal Clinical Microbiology 26:2279-2282

Summerbell, R.C. (2000). Form and function in the evolution of dermatophytes. En Biology of dermatophytes and other keratinolitic fungi. Rev. Iberoamericana de Micología, Editores Kushwaha \& J. Guarro.

Taplin,D.; Zaias, N.; Rebell, G. \& Blank, H. (1969). Isolation and recognition of dermatophytes on a new médium (DTM). Arch.Dermatol. 99:203-209

Vidotto V.; Moiraghi \& Ruggenini A. (1983). Influence of the substrate on culture and morphological characteristics of dermatophytes. Inicial results relating to strains isolated from 586 patients. G. Batteriol. Virol. Inmunol. 76: 118-126
Weitzman, I.; McGinnis, M. R.; Padhye, A. A. \& Ajello, L. (1986). The genus Arthroderma and its synonym Nannizzia. Mycotaxon 25:505-518

Weitzman, I. \& Summerbell, R.C. (1995). The dermatophytes. Clinical Microbiology Review 8:240-259

Young, N.C. (1972). Range of variation among isolates of Trichophyton rubrum. Sabouraudia 10:164-170

Zaror, L.; Moreno, M.I.; Vega, K.; Hering, M.; Frick O.P. (1995). Agentes de onicomicosis en manos y pies en Valdivia (Chile). Bol. Micol. 10:53-57

Zepgi, T.; Ma. S; Torres, M. C.; Silva, P. P. (2004). Onicomicosis en pacientes pediátricos. Rev. Chil. Dermatol. 20:199-203 